\title{
Repeat Selective Laser Trabeculoplasty for Glaucoma Patients: A Systematic Review and Meta-analysis
}

\author{
Hyunsoo J Jang ${ }^{1}$, Brian Yu², William Hodge ${ }^{3}$, Monali S Malvankar-Mehta ${ }^{4}$
}

\begin{abstract}
Aim and objective: To evaluate repeat selective laser trabeculoplasty (SLT) for treating primary open-angle glaucoma (POAG). Materials and methods: PubMed, CINAHL, and EMBASE were systematically searched along with grey literature. All English articles that measured intraocular pressure (IOP) before and after repeat SLT on adult patients with POAG were included. Studies were not filtered by location or publication date. Covidence was used to screen imported articles. Risk of bias assessment and data extraction was performed after screening. Meta-analysis was performed using STATA 16.0. Fixed-effect or random-effects models were developed depending on the presence of heterogeneity.

Results: Database and grey literature search identified 512 unique studies. After duplicate removal and screening, 12 articles were included and data from included studies were synthesized. Nine articles were included in the meta-analysis. Three studies were prospective observational studies, and nine studies were retrospective chart reviews. Due to the presence of heterogeneity, a random-effects model has been utilized that suggested significant IOP reduction (IOPR) by repeat SLT at 24 months follow-up.

Conclusion: Based on our results, repeat SLT could be an effective procedure in reducing IOP for patients with glaucoma for up to 24 months. Efficacy of third, fourth, or further SLT remains to be verified. More data from long-term, high-quality randomized-controlled trials (RCTs) are required to make conclusions.

Clinical significance: Repeat SLT may be an effective treatment for lowering IOP with minimal complications or safety issues. This may allow the use of SLT as a primary treatment for POGA, allowing the discontinuation of medications or eye drops and lead to additional benefits.

Keywords: Glaucoma, Intraocular pressure, Laser, Meta-analysis, Repeat selective laser trabeculoplasty, Selective laser trabeculoplasty, Systematic review, Trabeculoplasty.

Journal of Current Glaucoma Practice (2021): 10.5005/jp-journals-10078-1302
\end{abstract}

\section{INTRODUCTION}

Selective laser trabeculoplasty (SLT) as a first-line treatment has become a recent theme in the literature. Results of the LiGHT trial supported the change in clinical practice, citing lower cost and treatment burden associated with SLT when compared with medications and eye drops. ${ }^{1}$ In 1995, Latina introduced SLT as a procedure that uses a laser for selective perforation of pigmented cells in the trabecular meshwork (TM). ${ }^{2}$ The selective perforation increases fluid outflow from the eye and reduces intraocular pressure (IOP) while minimizing tissue damage. ${ }^{3,4}$ Since its approval by the FDA in 2001, SLT has emerged as one of the standard treatments for open-angle glaucoma. Selective laser trabeculoplasty is successful in about $80 \%$ of patients and can lower eye pressure by as much as $20-30 \%$, on average. ${ }^{5}$ While the use of medications and eye drops are also a common treatment option, poor patient compliance limits treatment success and toxicity presents risks to consider. ${ }^{6,7}$ The use of SLT as a primary treatment avoids these issues while also providing an effective IOP reduction (IOPR) and a modest cost-saving. .,9 $^{2}$

The prolonged IOPR with a one-time procedure of SLT can considerably reduce the treatment burden in many patients. However, in the long-term, IOPR by SLT may diminish in some patients, requiring further interventions. ${ }^{10}$ Fortunately, because the damage to the TM by SLT is minimal, a repeat procedure may be a safe option. Not only was repeat SLT shown to be similar in its efficacy to initial successful SLT treatment, but it was also shown to be effective even after the initial SLT failed to result in a sufficient IOP decrease. ${ }^{11-14}$

\begin{abstract}
1,2Department of Physiology and Pharmacology, Schulich School of Medicine and Dentistry, The University of Western Ontario, London, Ontario, Canada

${ }^{3}$ Department of Ophthalmology, Schulich School of Medicine and Dentistry, The University of Western Ontario, London, Ontario, Canada ${ }^{4}$ Department of Ophthalmology, Schulich School of Medicine and Dentistry, The University of Western Ontario, London, Ontario, Canada; Department of Epidemiology and Biostatistics, Schulich School of Medicine and Dentistry, The University of Western Ontario, London, Ontario, Canada
\end{abstract}

Corresponding Author: Monali S Malvankar-Mehta, Department of Ophthalmology, Schulich School of Medicine and Dentistry, The University of Western Ontario, London, Ontario, Canada; Department of Epidemiology and Biostatistics, Schulich School of Medicine and Dentistry, The University of Western Ontario, London, Ontario, Canada, Phone: +1 519-685-8500 ext. 61288, e-mail: monali.malvankar@sjhc. london.on.ca

How to cite this article: Jang HJ, Yu B, Hodge W, et al. Repeat Selective Laser Trabeculoplasty for Glaucoma Patients: A Systematic Review and Meta-analysis. J Curr Glaucoma Pract 2021;15(3):117-124.

Source of support: Nil

Conflict of interest: None

Together with recent developments in the literature supporting SLT as a first-line treatment, a conclusive report on the repeatability of SLT will further support a change in current ophthalmological practice. While there has been a recent abundance of research on the repeatability of SLT, a synthesis of findings has been lacking.

() The Author(s). 2021 Open Access This article is distributed under the terms of the Creative Commons Attribution 4.0 International License (https://creativecommons. org/licenses/by-nc/4.0/), which permits unrestricted use, distribution, and non-commercial reproduction in any medium, provided you give appropriate credit to the original author(s) and the source, provide a link to the Creative Commons license, and indicate if changes were made. The Creative Commons Public Domain Dedication waiver (http://creativecommons.org/publicdomain/zero/1.0/) applies to the data made available in this article, unless otherwise stated. 
Hence, this systematic review and meta-analysis are intended to synthesize current literature and evaluate repeated SLT for treating primary open-angle glaucoma (POAG).

\section{Materials and Methods}

\section{Search Strategy}

Database search was performed till May 2020 on PubMed, CINAHL, and EMBASE. Grey literature and conference abstracts from ARVO and AAO were searched through May 2020. Search terms were fabricated based on subject headings and keywords relating to "repeat selective laser trabeculoplasty" and "primary openangle glaucoma" with the assistance of an information specialist. Modifications were made to the search terms to fit the appropriate database-specific subject headings as necessary.

\section{Inclusion and Exclusion Criteria}

English articles that studied repeat SLT on patients with POAG were included. Studies that involved (1) repeat SLT, (2) measurement of IOP before and after repeated SLT, and (3) adults above the age of 18 years with POAG were included. Studies were not filtered by location or publication date.

\section{Screening}

The search results were imported into Covidence (Veritas Health Innovation, Melbourne, Australia) for the screening process. Citations from grey literature were first created using EndNote Basic then later imported into Covidence. The two-level screening was performed independently by two reviewers ( $\mathrm{HJ}$ and $\mathrm{BY})$. Titles and abstracts screening was performed in level 1 screening and full-text screening was performed in level 2 screening. In level 1 screening, all publications discussing repeat SLT were included. In level 2 screening, all publications involving repeat SLT, measurement of IOP before and after repeat SLT, and adults above the age of 18 years with POAG were included. Cohen's kappa coefficient $(\kappa)$ was calculated at each level of screening to assess the agreement of inclusion by the two reviewers. The kappa coefficient was 0.80 and 0.83 in level 1 and level 2 screening, respectively. Any conflicts at each level of screening were resolved through discussion.

\section{Quality Assessment}

Quality assessment of all articles that were included after level 2 screening was performed by the first author $(\mathrm{HJ})$ using the modified Downs and Black checklist. ${ }^{15}$ Articles were assessed on parameters such as reporting, internal and external validity, and power. A score out of 28 was given to each article. Studies were labeled based on their scores: articles with scores of 14 or less were labeled as poor quality, $15-29$ as fair quality, $20-25$ as good quality, and $26-28$ as excellent quality.

\section{Data Extraction}

Data extraction was performed by one reviewer (HJ). Data on study location and study design were collected including the number of study participants, number of participants that completed the study, number of eyes, average age, sex proportions, and proportion of patients with POAG. Further data were collected on the mean and standard deviation (SD) of IOP values from before and after the initial and repeat SLT, the time of IOP measurement, the number of participants measured in each interval, and any information regarding complications and side-effects related to initial and repeat SLT procedures.

\section{Statistical Analysis}

STATA 16.0 was used to perform the meta-analysis (STATA Corporation, College Station, TX, USA). The mean and SD of IOP measurements before and after the second SLT procedure were the main data of interest used to perform the meta-analysis. Range, confidence interval, standard error, and $p$ value were used to compute SD in studies where SD was missing. The mean IOPR were calculated as shown below: ${ }^{16}$

$$
\mathrm{IOPR}=10 \mathrm{P}_{\text {baseline }}-1 \mathrm{IOP} \text { endpoint }
$$

The mean IOPR values were then divided by the SD for each study to find the standard mean difference (SMD) to standardize the effect size across all studies. Each SMD value was then assigned with a weight that corresponded to the inverse of its variance to compute the summary effect.

The presence of heterogeneity was tested using the $l^{2}$ statistics, $Z$-value, and $\chi^{2}$ statistics. Additionally, in cases of significant heterogeneity (high $Z$-value, large $\chi^{2}$ value, and $p<0.01$ ), the DerSimonian and Laird method was used to calculate a randomeffects model. The results of the analysis were visualized using forest plots. A funnel plot was produced to check for publication bias.

\section{Publication Bias}

A funnel plot (Fig. 1) was produced and visually examined to identify the presence of publication bias. The plot shows included studies scattered along the central vertical line showing no signs of asymmetry. The small number of studies with high heterogeneity limits the assertion of publication bias being present in the study. Additionally, there may be other factors present that contribute to the patterns seen in the funnel plot.

\section{RESULTS}

\section{Study Characteristics}

Table 1 describes the patient characteristics of each study included in the meta-analysis. The searches identified 120 unique studies (Flowchart 1). The screening process ultimately resulted in 14 studies being synthesized. There were 3 prospective observational studies, 10 retrospective chart review studies, and 1 post hoc analysis of a randomized-controlled trial (RCT). Location of studies included

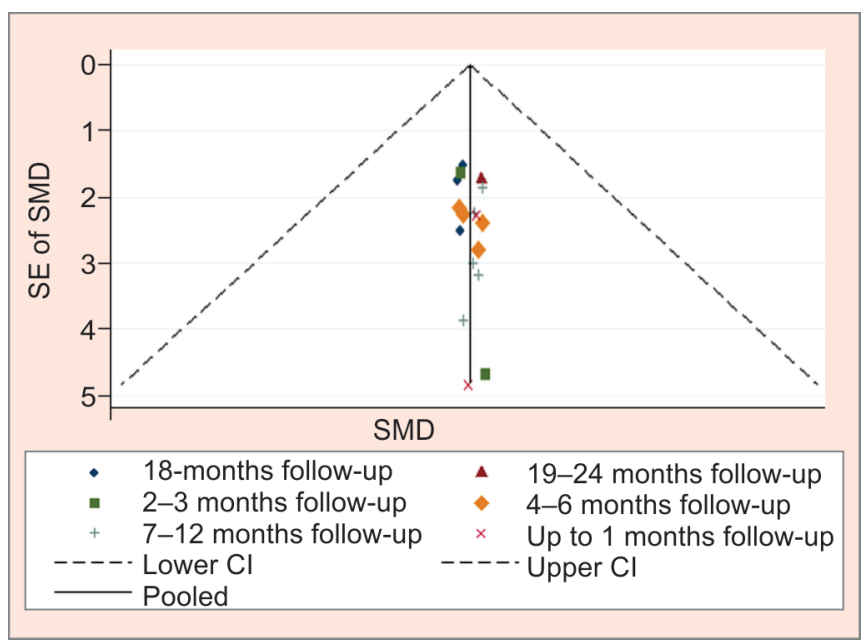

Fig. 1: Funnel plot on included studies on repeat SLT 
Table 1: Characteristics of studies included in the meta-analysis

\begin{tabular}{|c|c|c|c|c|c|c|c|c|c|}
\hline Studies & Location & Design & \# of eyes & Average Age & $\%$ POAG & $\%$ Female & Other treatments & $\begin{array}{l}\text { Treatment } \\
\text { angle }\end{array}$ & $\begin{array}{l}\text { \# of laser } \\
\text { shots }\end{array}$ \\
\hline $\begin{array}{l}\text { Avery et al. } \\
2013^{11}\end{array}$ & New Zealand & Retrospective & 42 & 61.1 & 100 & 48.8 & None & $360^{\circ}$ & $40-50$ \\
\hline $\begin{array}{l}\text { Copparam } \\
\text { et al. } 2019^{25}\end{array}$ & USA & Retrospective & 887 & 73.4 & 90 & 38 & - & $270^{\circ}-360^{\circ}$ & - \\
\hline $\begin{array}{l}\text { Durr et al. } \\
2016^{12}\end{array}$ & Canada & Retrospective & 38 & 70.2 & 71.1 & 65.79 & $\begin{array}{l}\text { Needling procedure (1 } \\
\text { patient) }\end{array}$ & $360^{\circ}$ & $40-60$ \\
\hline $\begin{array}{l}\text { Francis et al. } \\
2016^{13}\end{array}$ & USA & Retrospective & 137 & 72.5 & 80.3 & 61.3 & Medications & $360^{\circ}$ & $80-132$ \\
\hline $\begin{array}{l}\text { Garg et al. } \\
2020^{26}\end{array}$ & UK & Retrospective & 115 & 63.5 & 100 & 38 & None & $360^{\circ}$ & 100 \\
\hline $\begin{array}{l}\text { Hong et al. } \\
2009^{14}\end{array}$ & USA & Retrospective & 44 & 73.2 & 80 & 66 & - & $360^{\circ}$ & 102.1 \\
\hline $\begin{array}{l}\text { Khouri et al. } \\
2014 a^{23}\end{array}$ & USA & Retrospective & 51 & - & - & & Medications & $360^{\circ}$ & 102.6 \\
\hline $\begin{array}{l}\text { Peragallo } \\
\text { et al. } 2010^{24}\end{array}$ & USA & Retrospective & 19 & 69.7 & - & 35.3 & $\begin{array}{l}\text { Previous } \\
\text { trabeculectomy/ALT, } \\
\text { maximal tolerated } \\
\text { medical therapy }\end{array}$ & - & - \\
\hline $\begin{array}{l}\text { Polat et al. } \\
2016^{20}\end{array}$ & USA & Retrospective & 38 & 62.7 & 84.2 & 350 & $\begin{array}{l}\text { Maximal tolerated } \\
\text { medical therapy }\end{array}$ & $360^{\circ}$ & 100 \\
\hline
\end{tabular}

POAG, primary open-angle glaucoma; ALT, argon laser trabeculoplasty

Flowchart 1: PRISMA flow diagram of included studies

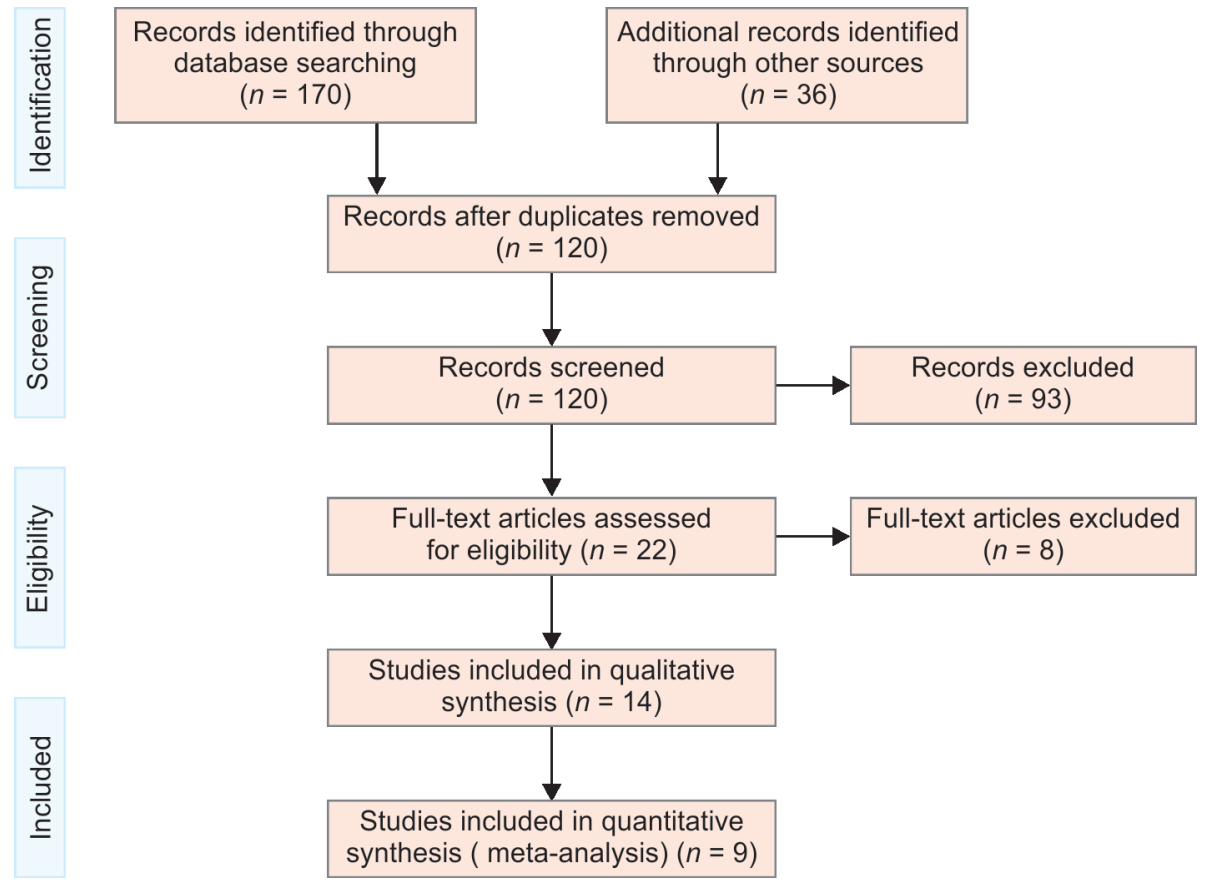

the United States, Canada, United Kingdom, Saint Lucia, and other Afro-Caribbean countries. Eight studies were of fair quality, and six studies were of poor quality according to the modified Downs and Black checklist.

\section{IOPR by Repeat SLT}

The IOP data from each study are summarized in Table 2. Results of meta-analysis indicate a significant reduction of IOP following a repeat SLT procedure across all follow-up period studied (Fig. 2). Significant heterogeneity was found between studies at 1-month, $<6$-month, and 12-month follow-up period $\left(l^{2}=86,92.3\right.$, and $94.5 \%$, respectively). Hence, a random-effects meta-analysis was performed using the DerSimonian and Laird method $(D+L)$ given significant heterogeneity among included articles. The studies collectively showed a significant reduction in IOP at 1-month follow-up (SMD = -0.96, Cl: $[-1.35,-0.57]), 2$ to 3 months follow-up 
@े

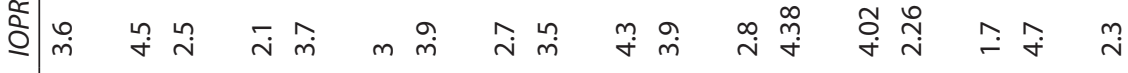

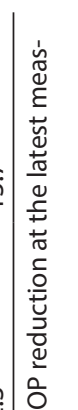

望

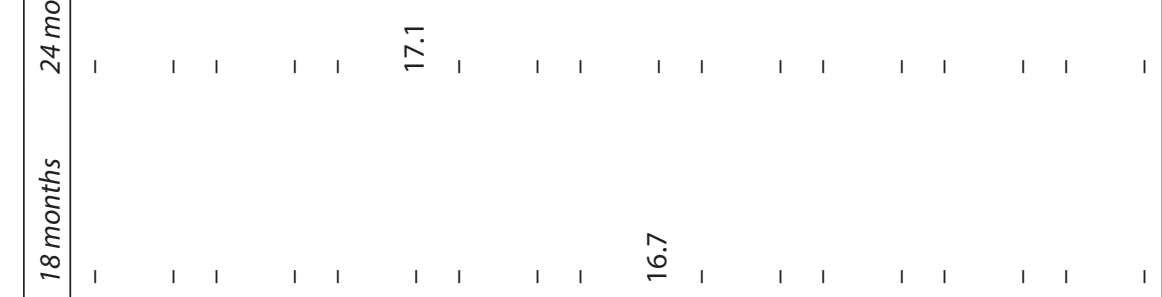

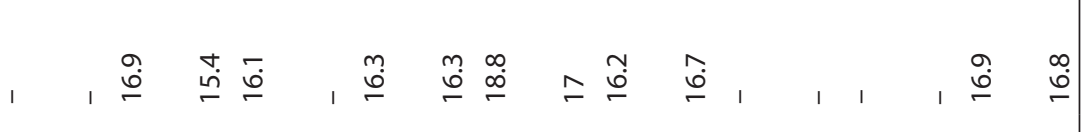

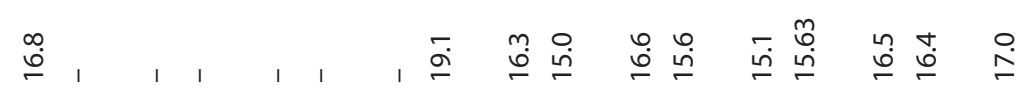

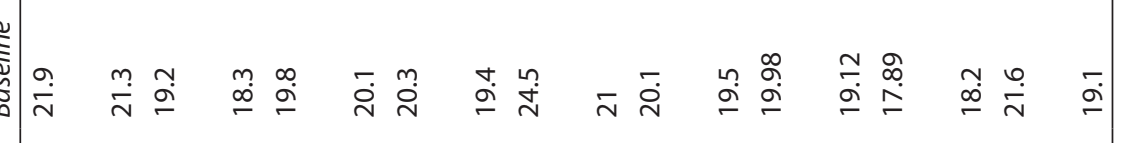

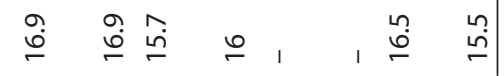

을 


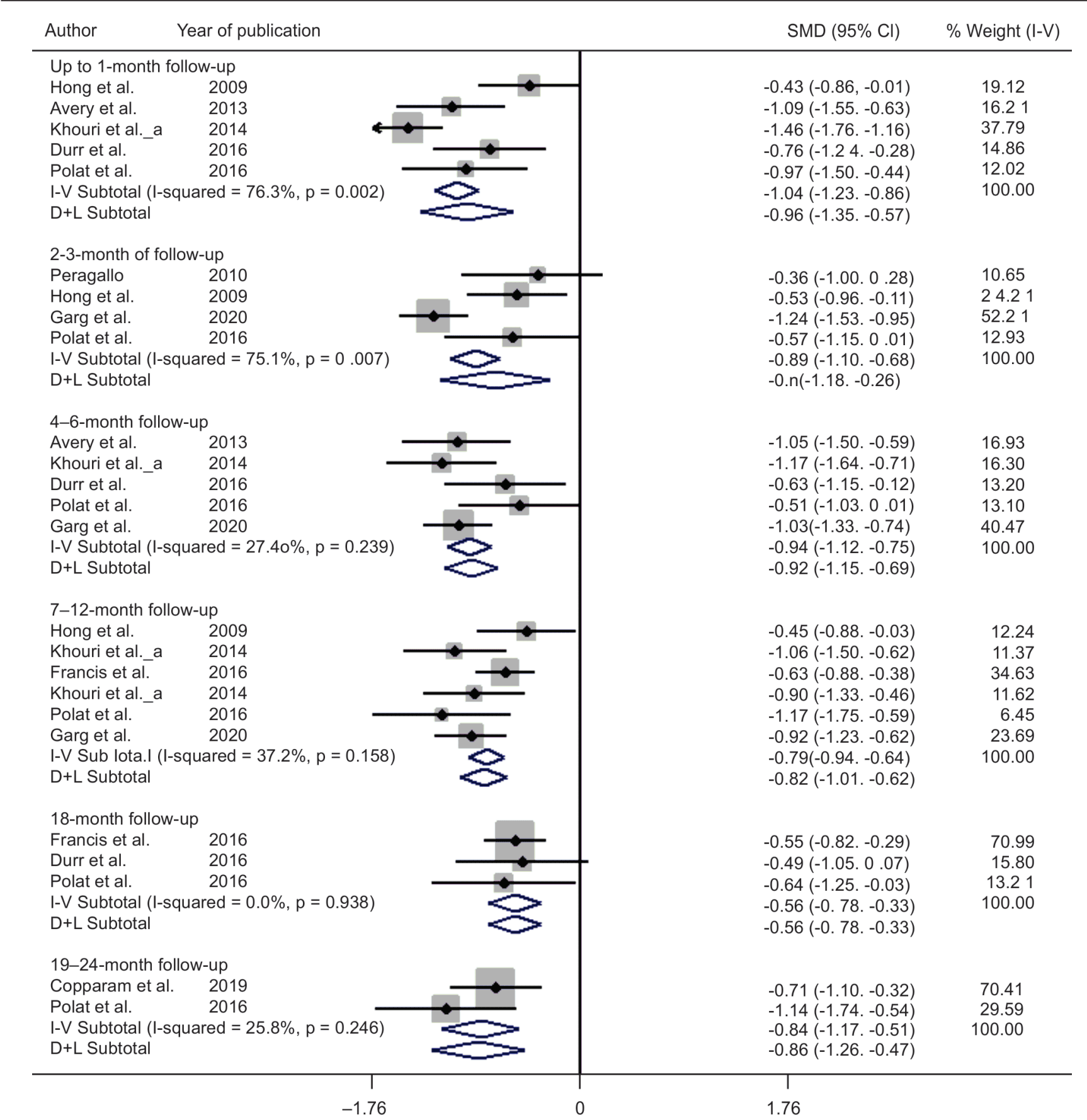

Fig. 2: Intraocular pressure reduction by repeat SLT in standardized mean difference (SMD) $(95 \% \mathrm{Cl})$

$(\mathrm{SMD}=-0.72, \mathrm{Cl}:[-1.18,-0.2]), 4-6$ months $(\mathrm{SMD}=-0.92, \mathrm{Cl}:$ $[-1.15,-0.69]), 7-12$ months $(\mathrm{SMD}=-0.82, \mathrm{Cl}=[-1.01,-0.62])$, 18 months (SMD $=-0.56, \mathrm{Cl}:[-0.78,-0.33])$, and 19-24 months $(\mathrm{SMD}=-0.86, \mathrm{Cl}:[-1.26,-0.47])$. Therefore, our results indicated a significant reduction in IOP after the repeat SLT procedure for up to 24 months.

\section{Efficacy of Third SLT}

Effects of the third SLT were studied by Avery et al., Realini and Curtis, Lai and Bournias, and Khouri et al. ${ }^{11,17-19}$ Khouri et al. reported that the third SLT remained effective for up to 18 months (mean IOP was 16.6 and $18.5 \mathrm{~mm} \mathrm{Hg}$ at 18 and 24 months after the third
SLT, respectively). ${ }^{19}$ Avery et al. reported a success rate of $56 \%$ with SLT3 (defined as IOPR of $20 \%$ or greater from pre-SLT3 IOP) which was comparable to that of 55 and $66 \%$ of the initial SLT and repeat SLT, respectively. ${ }^{11}$ Realini and Curtis reported that all treatments with SLT3 remained successful at the time with a mean IOP of 12.8 $\mathrm{mm} \mathrm{Hg} .^{17}$ Meanwhile, Lai and Bournias reported no success with any third SLT procedure: none of the five eyes sustained an IOP decrease of $\geq 5 \mathrm{~mm} \mathrm{Hg}$ for 1 year. ${ }^{18}$

\section{Complications}

No studies reported any major complications associated with either initial or repeat SLT. Avery et al. reported mild discomfort (18\% of 
study participants), headache (4\%), and photophobia (3\%). ${ }^{11}$ Hong et al. reported IOP spike and conjunctival hyperemia after both initial and repeat SLT as well as mild anterior uveitis (two eyes) after initial SLT. ${ }^{14}$ Realini and Curtis reported that moderate photophobia occurred and resolved within 2-3 days in most patients without treatment. $^{17}$

\section{Discussion}

The results of the meta-analysis and the existing literature suggest that repeat SLT may lead to significant IOPR up to 24 months after the procedure. Studies also report that the IOPR by repeat SLT tends to remain for a longer duration compared with initial SLT, even if this effect is non-significant. Durr and Harasymowycz reported a $10.3 \%$ reduction in IOP 15 months after repeat SLT compared with $8.7 \%$ after the first SLT. ${ }^{12}$ Likewise, Avery et al. also reported a nonsignificant increase in the duration of IOPR after repeat SLT (13.1 months for repeat SLT vs 6.9 months for SLT1). ${ }^{11}$ Similar trends were also seen by Polat et al. ${ }^{20}$ who reported a median Kaplan-Meier survival time of 570 days for the initial SLT vs 1,054 days for the repeat SLT. While most studies in the review reported no serious side effects of repeat SLT, Baser and Akbulut presented two rare cases of peripheral anterior synechiae (PAS) after repeat SLT. ${ }^{20}$ This is the only report of PAS after repeat SLT, and the relationship between repeat SLT and PAS remains unclear.

There were few studies on the effects of third SLT. While most studies report success with third SLT, Lai and Bournias suggest the contrary. ${ }^{11,17-19}$ Furthermore, the limited number of samples was an issue with most studies on third SLT: Avery et al. had 9 eyes, Realini and Curtis had 5, Khouri et al. had 14, and Lai and Bournias had 5 eyes that went through the third SLT procedure. ${ }^{11,17-19}$ Additionally, any complications or safety concerns related to the third SLT remain unreported.

Repeat SLT could be an effective treatment option for lowering IOP. Its IOP-reducing effect is significant, and its side effects are minimal. This is especially important when other treatments, such as drugs and eye drops, involve issues with side effects, patient compliance, and cost. Repeat SLT may allow some patients to discontinue the use of drugs or eye drops and eliminate these concerns. However, the effects of repeat SLT may not be permanent, and patients may require an additional SLT procedure or the resumption of pharmacological treatments to lower IOP as the IOP rises back again. Third SLT may be effective until 24 months after the procedure; however, current studies are limited in sample size and show a tapering IOP-reducing effect. ${ }^{11}$ Furthermore, the potential consequences of continued repetition of SLT treatments remain unknown. Hence, while a third SLT may also be a valid treatment option, more results from long-term, high-quality studies are required to make conclusions.

The limited availability of the literature on repeat SLT limits the conclusions of the current research study. Most studies included were retrospective reviews with a few being prospective studies. Therefore, additional data from high-quality, long-term RCTs are required in the future. No studies involved randomizations or control groups; therefore, if such studies are conducted in the future, a systematic review including those good quality articles will be useful to make strong conclusions. Furthermore, few studies included a follow-up period of $>15$ months. Thus, studies with a long-term follow-up period would be critical. Lastly, the high heterogeneity between the included studies necessitated the use of a random-effects model. Differences in technique, surgeon's experience, and SLT facilities may contribute to the substantial heterogeneity between the studies. Additional factors that may introduce heterogeneity include different patient characteristics, demographics, inclusion/exclusion criteria, concurrent IOPreducing treatments, as well as different locations and time-period of the studies.

In conclusion, repeat SLT may be effective for long-term IOPR with minimal complications or safety issues. It may also allow for the discontinuation of medications or eye drops which may lead to added benefits. While its use as a primary treatment option for POAG seems promising, more research on the topic is needed to alleviate any residual safety concerns.

\section{Clinical Significance}

Repeat SLT may be an effective treatment for long-term IOPR with minimal complications or safety issues. This may allow the use of SLT as a primary treatment for POAG, allowing the discontinuation of medications or eye drops and lead to additional benefits.

\section{References}

1. Gazzard G, Konstantakopoulou E, Garway-Heath D, et al. Selective laser trabeculoplasty versus eye drops for first-line treatment of ocular hypertension and glaucoma (LiGHT): a multicentre randomised controlled trial. Lancet 2019;393(10180):1505-1516. DOI: 10.1016/S0140-6736(18)32213-X.

2. Noecker RJ. How SLT works. Glaucoma Today 2005. 30-31. http:// glaucomatoday.com/pdfs/0305_01.pdf (accessed October 25, 2019).

3. Latina MA, Park C. Selective targeting of trabecular meshwork cells: In vitro studies of pulsed and CW laser interactions. Exp Eye Res 1995;60(4):359-371. DOI: 10.1016/s0014-4835(05)80093-4.

4. Latina MA, Sibayan SA, Shin DH, et al. Q-switched 532-nm Nd:YAG laser trabeculoplasty (selective laser trabeculoplasty). Ophthalmology 1998;105(11):2082-2090. DOI: 10.1016/s0161-6420(98)91129-0.

5. Katz LJ, Lee D, Zheng C. Selective Laser Trabeculoplasty as Primary Glaucoma Therapy. Glaucoma Res Found 2018. https://www. glaucoma.org/treatment/selective-laser-trabeculoplasty-asprimary-glaucoma-therapy.php (accessed September 11, 2019).

6. Reardon G, Kotak S, Schwartz GF. Objective assessment of compliance and persistence among patients treated for glaucoma and ocular hypertension: a systematic review. Patient Prefer Adherence 2011;5:441-463. DOI: 10.2147/PPA.S23780.

7. Robciuc A, Witos J, Ruokonen SK, et al. Pure glaucoma drugs are toxic to immortalized human corneal epithelial cells, but they do not destabilize lipid membranes. Cornea 2017;36(10):1249-1255. DOI: 10.1097/ICO.0000000000001322.

8. Melamed S, Simon GJBen, Levkovitch-Verbin H. Selective laser trabeculoplasty as primary treatment for open-angle glaucoma. Arch Ophthalmol 2003;121(7):957-960. DOI: 10.1001/archopht.121.7.957.

9. Lee R, Hutnik CML. Projected cost comparison of selective laser trabeculoplasty versus glaucoma medication in the Ontario health insurance plan. Can J Ophthalmol 2006;41(4):449-456. DOI: 10.1016/ S0008-4182(06)80006-2.

10. Juzych MS. Comparison of long-term outcomes of selective laser trabeculoplasty versus argon laser trabeculoplasty in open-angle glaucoma. Ophthalmology 2004;111(10):1853-1859. DOI: 10.1016/j. ophtha.2004.04.030.

11. Avery N, Soon G, Simon A. Repeatability of primary selective laser trabeculoplasty in patients with primary open-angle glaucoma. Int Ophthalmol 2013(5):501-506. DOI: 10.1007/s10792-013-9729-3.

12. Durr GM, Harasymowycz P. The effect of repeat 360 -degree selective laser trabeculoplasty on intraocular pressure control in openangle glaucoma. J Fr Ophtalmol 2016;39(3):261-264. DOI: 10.1016/j. jfo.2015.10.008. 
13. Francis BA, Loewen N, Hong B, et al. Repeatability of selective laser trabeculoplasty for open-angle glaucoma. BMC Ophthalmol 2016;16(1):128. DOI: 10.1186/s12886-016-0299-9.

14. Hong BK, Winer JC, Martone JF, et al. Repeat selective laser trabeculoplasty. J Glaucoma 2009;18(3):180-183. DOI: 10.1097/ IJG.0b013e31817eee0b.

15. Downs SH, Black N. The feasibility of creating a checklist for the assessment of the methodological quality both of randomised and non-randomised studies of health care interventions. J Epidemiol Community Health 1998;52(6):377-384. DOI: 10.1136/jech.52. 6.377.

16. Zhang WY, Po ALW, Dua HS, et al. Meta-analysis of randomised controlled trials comparing latanoprost with timolol in the treatment of patients with open angle glaucoma or ocular hypertension. Br J Ophthalmol 2001;85(8):983-990. DOI: 10.1136/bjo.85. 8.983.

17. Realini T, Curtis H. Repeat selective laser trabeculoplasty in AfroCaribbean eyes with primary open-angle glaucoma. Investigative Ophthalmology \& Visual Science, Vol. 57, 2016 ARVO Annu. Meet., Seattle, Washington: The Association for Research in Vision and Ophthalmology; 2016. https://iovs.arvojournals.org/article. aspx?articleid $=2562518$.

18. Lai J, Bournias TE. Repeatability of selective laser trabeculoplasty (SLT). Investigative Ophthalmology \& Visual Science, Vol 46, J. Lai; T.E. Bournias. 2005 ARVO Annu. Meet., Fort Lauderdale, Florida: The Association for Research in Vision and Ophthalmology; 2005, p. 119.
19. Khouri AS, Berezina T, Maltzman BA, et al. Efficacy of second and third repeat selective laser trabeculoplasty. AAO 2010. Chicago, Illinois: American Academy of Ophthalmology, 2010.

20. Polat J, Grantham L, Mitchell K, et al. Repeatability of selective laser trabeculoplasty. Br J Ophthalmol 2016;100:1437-1441.

21. Baser EF, Akbulut D. Significant peripheral anterior synechiae after repeat selective laser trabeculoplasty. Can J Ophthalmol 2015;50(3):e36-e38. DOI: 10.1016/j.jcjo.2015.02.006.

22. Khouri AS, Lari HB, Berezina $T L$, et al. Original article long term efficacy of repeat selective laser trabeculoplasty. J Ophthalmic Vis Res 2014;9:444-448.

23. Khouri AS, Lin J, Berezina $T L$, et al. Repeat selective laser trabeculoplasty can be effective in eyes with initial modest response. Middle East Afr J Ophthalmol 2014;21:205-209.

24. Peragallo JH, Malen M, Lai PC. Effectiveness of repeat selective laser trabeculoplasty on primary open angle glaucoma (POAG) patients. 2010 ARVO Annu Meet. Fort Lauderdale, Florida: The Association for Research in Vision and Ophthalmology; 2010. p. 4427.

25. Copparam S, Kuley B, Zheng CX, et al. Outcomes of repeat selective laser trabeculoplasty for open-angle glaucoma. 2019 ARVO Annu Meet. Vancouver, British Columbia; 2019.

26. Garg A, Vickerstaff V, Nathwani N, et al. Efficacy of repeat selective laser trabeculoplasty in medication-naive open-angle glaucoma and ocular hypertension during the LiGHT trial. Ophthalmology [Internet] 2020;127:467-476. Available from: https://doi.org/10.1016/j. ophtha.2019.10.023. 


\section{Supplementary Materials \\ Search Strategy \\ MEDLINE (14 May 2020)}

1 Glaucoma/or Glaucoma, Open-Angle/or glaucoma.mp. or open-angle glaucoma.mp. or open angle glaucoma.mp. or 70,272 primary open-angle glaucoma.mp. or primary open angle glaucoma.mp. or OAG.mp. or POAG.mp. [mp = title, abstract, original title, name of substance word, subject heading word, floating sub-heading word, keyword heading word, organism supplementary concept word, protocol supplementary concept word, rare disease supplementary concept word, unique identifier, synonyms]

2 (selective laser trabeculoplasty or SLT or RSLT or repeat* SLT or repeat* selective laser trabeculoplasty).mp. [mp $=$ title, abstract, original title, name of substance word, subject heading word, floating sub-heading word, keyword heading word, organism supplementary concept word, protocol supplementary concept word, rare disease supplementary concept word, unique identifier, synonyms]

3 Repeat*

EMBASE (14 May 2020)

1 Glaucoma/or open angle glaucoma/or glaucoma.mp. or open-angle glaucoma.mp. or open angle glaucoma.mp. or primary open-angle glaucoma.mp. or primary open angle glaucoma.mp. or OAG.mp. or POAG.mp. [mp = title, abstract, heading word, drug trade name, original title, device manufacturer, drug manufacturer, device trade name, keyword, floating subheading word, candidate term word]

2 (selective laser trabeculoplasty or SLT or RSLT or repeat* SLT or repeat* selective laser trabeculoplasty).mp. [mp $=$ title, abstract, heading word, drug trade name, original title, device manufacturer, drug manufacturer, device trade name, keyword, floating subheading word, candidate term word]

3 Repeat*

$3 \quad 1$ and 2 and 3

CINAHL (14 May 2020)

1 (MH "Glaucoma") OR "glaucoma" or "open-angle glaucoma" or "open angle glaucoma" or "primary open-angle glaucoma" 10,506 or "primary open angle glaucoma" or "OAG" or "POAG"

2 Selective laser trabeculoplasty or slt or rslt or repeat slt or repeat selective laser trabeculoplasty

ARVO (14 May 2020)

Repeat* "selective laser trabeculoplasty" AND "primary open-angle glaucoma"

AAO (14 May 2020) 